\title{
Avaliação da atividade proliferativa celular da leucoplasia bucal por meio do método AgNOR
}

\section{Assessment of cell proliferative activity of oral leukoplakia through the AgNOR method}

\author{
Angélica Zanata* \\ Carmen Silvia Busin* \\ Soluete Oliveira da Silva** \\ Maria Salete Sandini Linden ${ }^{* * * *}$ \\ Micheline Sandini Trentin ${ }^{* * * * *}$ \\ João Paulo De Carli ${ }^{* * *+*}$
}

\section{Resumo}

A leucoplasia é considerada uma importante lesão bucal cancerizável. Objetivo: realizar um estudo epidemiológico dos casos de leucoplasia bucal registrados no Serviço de Diagnóstico Histopatológico do Instituto de Ciências Biológicas da Universidade de Passo Fundo (SDH/ICB-UPF) entre 1989 e 2012, aplicando a tais casos o método AgNOR para avaliar a atividade proliferativa celular desses. Materiais e método: o trabaIho consistiu num estudo epidemiológico-histoquímico transversal de 44 casos de leucoplasia. Após a confirmação diagnóstica e a tabulação dos dados clínicos e histopatológicos dos casos de leucoplasia, novas secções histológicas $(3 \mu \mathrm{m})$ das lesões foram impregnadas pela prata por meio do método AgNOR. As lâminas obtidas foram fotografadas em imersão, tendo sido contabilizadas as NORs de 100 células de cada caso. Tal procedimento foi realizado com o auxílio do software Image $\mathrm{Too}^{\circledR}$. Os resultados foram comparados levando-se em conta as diferentes características clínicas e histopatológicas dos casos de leucoplasia. Resultados: a faixa etária mais frequente foi de 61 a 76 anos (19 casos - 43,2\%), 22 pacientes pertenciam ao sexo masculino (50\%) e a etnia predominante foi a leucoderma (36 casos $-81,8 \%$ ). No que diz respeito aos hábitos nocivos, 23 pacientes $(52,3 \%)$ informaram ser tabagistas. Ao se comparar o número médio de NORs dos casos de leucoplasia a diferentes características clínicas das lesões, não foi observada uma relação estatisticamente significativa. Conclusão: conclui-se que a leucoplasia acomete principalmente homens leucodermas, fumantes e com mais de 60 anos de idade. Além disso, na amostra ora estudada, a relação da atividade proliferativa celular da leucoplasia bucal com as características clínicas/ histopatológicas das lesões não foi estatisticamente significativa.

Palavras-chave: Leucoplasia oral. AgNOR. Actividad proliferative.

\section{Introdução}

A leucoplasia é a lesão bucal cancerizável mais frequente, é definida, segundo a Organização Mundial de Saúde $(\mathrm{OMS})^{1}$, como uma mancha ou placa branca não removível à raspagem e que não pode ser classificada clínica ou histopatologicamente como outra enfermidade. Lesões em língua, vermelhão dos lábios e assoalho bucal somam mais de $90 \%$ daquelas que exibem displasia ou carcinoma. ${ }^{2}$ Em diversos estudos e localizações, a taxa de transformação da leucoplasia em lesão maligna varia entre 0,6 e $18 \%^{3}$.

\section{http://dx.doi.org/10.5335/rfo.v20i1.3955}

Cirurgiã-dentista pela Faculdade de Odontologia da UPF.

Doutora em Biologia Celular, Professora do Instituto de Ciências Biológicas da UPF.

Especialista em Patologia Bucal, Doutora em Odontologia - Área de Estomatologia Clínica; Professora da Faculdade de Odontologia da UPF.

Especialista em Implantodontia, Doutora Implantodontia - Professora da Faculdade de Odontologia da UPF.

Especialista em Implantodontia, Doutora Implantodontia - Professora da Faculdade de Odontologia da UPF.

Especialista em Prótese Dentária, Mestre e Doutor em Odontologia - Área de Estomatologia; Professor da Faculdade de Odontologia da UPF. 
No intuito de avaliar o potencial de transformação maligna das leucoplasias, vários autores têm estudado diferentes marcadores de proliferação celular, entre eles a técnica de Regiões Argirofílicas Organizadoras Nucleolares (AgNOR), a qual possibilita a visualização das regiões organizadoras nucleolares (NORs) ativas durante a interfase por meio da impregnação pela prata ${ }^{4}$.

As NORs ativas são associadas com proteínas acídicas, argirofílicas não histônicas, que são visualizadas pelo método AgNOR. No DNA humano, as NORs se localizam no braço curto dos cromossomos acrocêntricos 13, 14, 15, 21 e 22. Diferentes estudos observaram aumento gradual da média de pontos de AgNOR por núcleo na medida em que o epitélio bucal normal sofre alterações de maturação até sua progressão maligna ${ }^{4,5}$.

Em qualquer estágio do ciclo celular, o número de NORs parece inversamente proporcional ao tempo desse ciclo, ou seja, quanto maior a quantidade de NORs, mais curto será o ciclo celular e maior o índice de divisão das células.

O objetivo do presente estudo é realizar um levantamento epidemiológico dos casos de leucoplasia bucal registrados no Serviço de Diagnóstico Histopatológico do ICB/UPF, aplicando a tais casos o método AgNOR, a fim de relacionar a atividade proliferativa celular desses com suas características clínicas/histopatológicas.

\section{Materiais e método}

O presente trabalho foi aprovado pelo Comitê de Ética em pesquisa da UPF (parecer no $172 / 2011$ ) e consiste num estudo epidemiológico-histoquímico transversal. Foram analisados 44 casos de leucoplasia bucal diagnosticados histopatologicamente entre os anos de 1989 e 2012 no Serviço de Diagnóstico Histopatológico do Instituto de Ciências Biológicas da Universidade de Passo Fundo (ICB/UPF). Os dados epidemiológicos referentes aos pacientes e respectivas lesões foram obtidos a partir dos relatórios histopatológicos dos casos de leucoplasia bucal e tabulados em planilha eletrônica Excel ${ }^{\circledR}$. Previamente à seleção dos casos, todas as lâminas histológicas coradas com hematoxilina-eosina de biópsias incisionais ou excisionais foram revistas por um patologista oral para confirmar o diagnóstico.

Posteriormente, foi realizado um estudo histoquímico transversal dos casos de leucoplasia. Para isso, foram obtidas secções de $3 \mu \mathrm{m}$ de blocos teciduais incorporados com parafina e realizada a impregnação tecidual pelo método AgNOR de acordo com o protocolo descrito por Nunes et al. (1991)6.

As lâminas foram fotografadas em imersão, ampliadas em 1000X, tendo sido capturadas microfotografias de 100 células de cada caso, escolhidas aleatoriamente. As NORs de tais células foram contabilizadas com a ajuda do software Image Tool ${ }^{\circledR}$
(Department of Dental Diagnostic Science at The University of Texas Health Science Center, San Antonio, Texas, EUA) e a análise estatística foi realizada por meio do teste ANOVA.

\section{Resultados}

Na Figura 1, observa-se o número de casos de leucoplasia registrados no Serviço de Diagnóstico Histopatológico do ICB/UPF no período compreendido pelo presente estudo (1989 e 2012) em relação ao gênero.

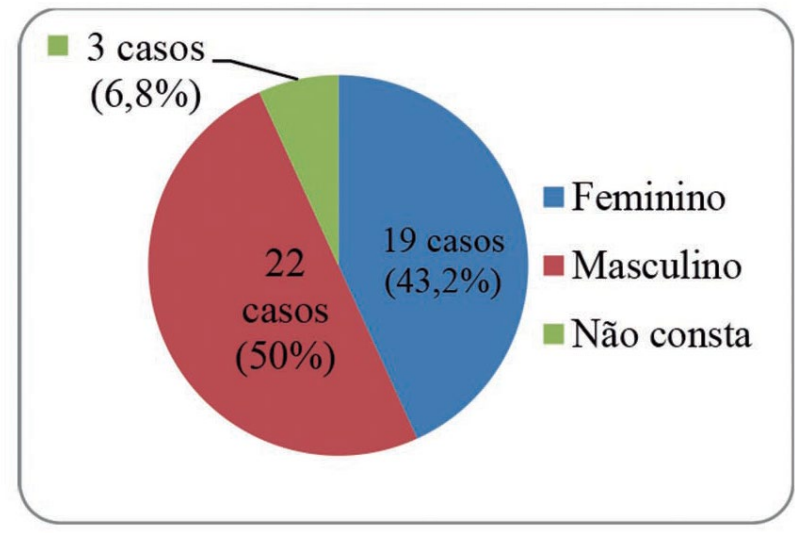

Figura 1 - Distribuição dos casos de leucoplasia bucal quanto ao gênero dos pacientes (ICB/UPF 1989-2012)

Em relação à idade dos pacientes, observou-se uma variação entre 30 e 76 anos, com uma idade média de 55 anos. A faixa etária mais atingida esteve entre 61 e 76 anos, com 19 casos (43,2\%), (Figura 2).

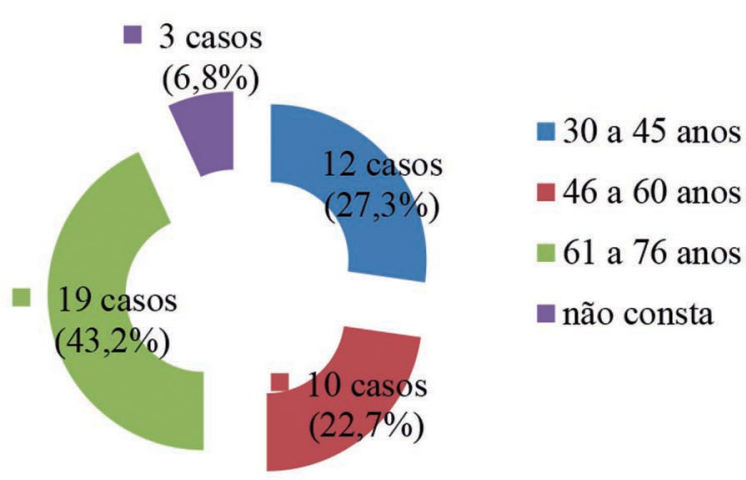

Figura 2 - Distribuição dos casos de leucoplasia bucal quanto à idade dos pacientes (ICB/UPF 1989-2012)

Quanto aos hábitos deletérios relatados pelos pacientes, o mais frequente foi o fumo (23 casos - 52,3\%). Em quinze casos $(34,1 \%)$, não houve uma causa específica ou o paciente não soube informar. A exposição solar apresentou-se em 2 casos $(4,5 \%)$, o fumo e a exposição solar associados em 3 casos $(6,8 \%)$ e o trauma por mordida e o fumo associados em 1 caso $(2,3 \%)$. 
Quanto à lesão fundamental, tinta casos $(68,2 \%)$ apresentaram-se sob a forma de placa, seis casos $(13,6 \%)$ apresentaram-se sob a forma de mancha e oito laudos $(18,2 \%)$ não apresentavam tal informação.

Em relação ao tamanho da lesão, quinze casos $(34,09 \%)$ apresentaram mais do que $20 \mathrm{~mm}$ no seu maior diâmetro, 26 casos $(59,09 \%)$ apresentaram menos do que $20 \mathrm{~mm}$ e três casos $(6,81 \%)$ não apresentavam essa informação.

Observou-se que a maior parte das lesões era assintomática (27 casos - 61,4\%), doze casos $(27,3 \%)$ apresentavam ardência, dois casos $(4,5 \%)$ revelaram dor, um caso revelou dor e ardência associadas $(2,3 \%)$ e dois laudos (4,5\%) não apresentavam esse dado.

Quanto à localização, a língua foi a parte mais acometida, com dez casos $(22,7 \%)$, seguida da mucosa jugal e rebordo alveolar, ambos com oito casos $(18,2 \%)$, e mucosa labial, com seis casos $(13,6 \%)$. As demais regiões representaram $27,4 \%$ dos casos (área retromolar - quatro casos, palato mole - quatro casos, assoalho de boca - um caso, palato duro - um caso, área retromolar e rebordo alveolar - um caso, rebordo alveolar e assoalho de boca - um caso) (Figura 3).

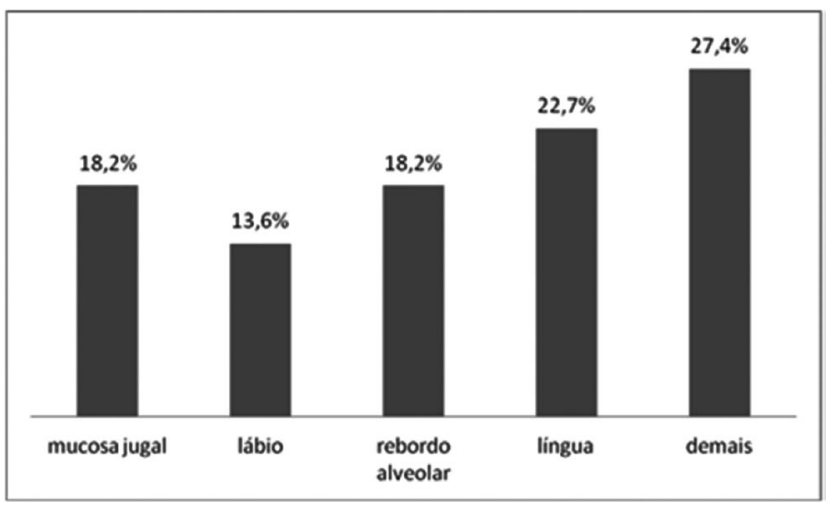

Figura 3 - Distribuição dos casos de leucoplasia bucal quanto à localização da lesão (ICB/UPF 1989-2012)

Quanto à consistência da lesão, vinte e três casos $(52,3 \%)$ eram firmes, dez casos $(22,7 \%)$ eram moles, três casos $(6,8 \%)$ apresentaram consistência elástica e oito laudos $(18,2 \%)$ não apresentaram este dado.

Em relação à superfície, a mais prevalente foi a rugosa (24 casos $-54,5 \%$ ), em seguida foi a plana (onze casos - 25\%), a ulcerada se mostrou presente em 2 casos $(4,5 \%)$, um caso apresentou superfície rugosa e ulcerada associadas (2,3\%), um caso apresentou superfície plana e ulcerada associadas $(2,3 \%)$. Em cinco casos $(11,4 \%)$, os laudos não continham informação sobre a superfície.

Em relação aos bordos das lesões, em treze casos $(29,5 \%)$ foram definidos, em doze casos $(27,3 \%)$ indefinidos, e em dezenove laudos $(43,2 \%)$ não se obteve tal informação.

Quanto à coloração, em trinta e três casos (75\%) as lesões eram brancas ou claras, em sete casos $(15,9 \%)$ eram brancas e avermelhadas, em dois ca- sos $(4,5 \%)$ eram brancas e arroxeadas, em um caso $(2,3 \%)$ a leucoplasia era escura ou enegrecida e em um laudo $(2,3 \%)$ não se obteve esta informação.

Com relação às características histopatológicas, a acantose associada à hiperceratose/hiperparaceratose/hiperortoceratose foi predominante, com 28 casos $(63,6 \%)$. Somente a acantose esteve presente em um caso $(2,3 \%)$ e somente a displasia epitelial manifestou-se em três casos $(6,8 \%)$. Os demais casos apresentavam uma associação de hiperceratose/ hiperparaceratose/hiperortoceratose, displasia epitelial acantose e papilomatose.

Ao se relacionar o número médio de NORs das lesões com cada uma de suas características clínicas ou histopatológicas, notou-se não haver significância estatística (Tabela 1).

Tabela 1 - Características clínicas ou histopatológicas das lesões de leucoplasia estudadas e a respectiva significância estatística com o no médio de NORs, obtido pela média das NORs de 100 núcleos celulares de cada lesão

\begin{tabular}{|c|c|}
\hline $\begin{array}{c}\text { Característica clínica ou } \\
\text { histopatológica }\end{array}$ & $\begin{array}{c}\text { Significância estatística } \\
\text { obtida, quando cruzado } \\
\text { com o n⿳0 médio de NORs } \\
(p)\end{array}$ \\
\hline Idade & 0,384 \\
\hline Gênero & 0,894 \\
\hline Etnia & 0,961 \\
\hline Hábitos deletérios & 0,788 \\
\hline Tempo de evolução & 0,831 \\
\hline Lesão fundamental & 0,316 \\
\hline Sintomatologia & 0,260 \\
\hline Localização & 0,290 \\
\hline Consistência & 0,537 \\
\hline Superfície & 0,662 \\
\hline Bordos & 0,855 \\
\hline Coloração & 0,480 \\
\hline $\begin{array}{l}\text { Características } \\
\text { histopatológicas }\end{array}$ & 0,060 \\
\hline
\end{tabular}

\section{Discussão}

O presente estudo buscou avaliar as características clínicas, histopatológicas e a atividade proliferativa celular de uma amostra de 44 casos de leucoplasia bucal, realizando uma comparação com os dados disponíveis na literatura.

A observação de que as leucoplasias bucais são mais comuns no gênero masculino do que no feminino neste estudo $(1,15: 1)$ é consistente com a maior parte dos relatos da literatura, provavelmente devido à associação com o hábito do tabagismo ${ }^{7}$. No entanto, estudo recente indica uma tendência para 
que a diferença de frequência de leucoplasias bucais entre os gêneros diminua, uma vez que o tabagismo torna-se cada vez mais comum entre as mulheres ${ }^{8}$.

O estudo de Vázquez-Álvarez et al. ${ }^{8}$ (2010) revisou os prontuários de 54 pacientes na unidade ligada ao curso de Mestrado em Medicina Oral, Cirurgia Oral e Implantologia da Universidade de Santiago de Compostela, na Espanha, e relatou que a faixa etária mais frequente em ambos os gêneros foi a sexta década de vida. Num estudo realizado na Índia, em um universo de 2920 participantes, a faixa etária mais frequente para o diagnóstico da leucoplasia bucal foi de 21 a 30 anos $^{9}$. No presente estudo, a idade média dos pacientes foi de 55 anos e a faixa etária mais atingida foi de 61 a 76 anos, acometendo $43,2 \%$ dos casos ( $n=19$ ). Nota-se assim, haver uma discrepância significativa entre as faixas etárias dos pacientes de diferentes estudos. Tal resultado pode ser devido aos diferentes hábitos de vida, em diferentes regiões geográficas (fundamentalmente o tabagismo, que é altamente prevalente na Índia) ou pode ser, também, consequência dos critérios de seleção das amostras de pacientes estudadas.

Neste estudo, o fumo foi o hábito deletério mais frequente (vinte e três casos - 52,3\%). Corroborando nosso resultado, o hábito de fumar tabaco aparece em todos os artigos revisados como sendo o principal fator associado ao desenvolvimento da leucoplasia bucal, indicando que mais de $80 \%$ dos pacientes com esse tipo de lesão são fumantes. Os fumantes descompensados apresentam um número maior de lesões, as quais são também maiores do que aquelas encontradas em pacientes que fumam pouco. Além disso, uma grande proporção das leucoplasias bucais desapareceu, ou diminuiu, após um ano do hábito de fumar ter sido cessado ${ }^{3,10,11}$.

Em $34,1 \%$ da amostra deste estudo (quize casos), os fatores etiológicos foram inespecíficos ou o paciente não soube informar qual foi a causa da leucoplasia e em $4,5 \%$ dos casos (dois casos), a causa foi a exposição solar. A radiação ultravioleta também é um fator etiológico da leucoplasia, quando essa aparece no vermelhão do lábio inferior, normalmente confundida com queilite actínica ${ }^{2}$.

No presente estudo, a língua foi o sítio anatômico mais acometido pela leucoplasia bucal, com $22,7 \%$ (dez casos). Em outro estudo, o bordo lateral da língua também foi o local mais frequente da leucoplasia (25,9\% dos pacientes) $)^{8}$. A mucosa jugal e as comissuras labiais são envolvidas mais frequentemente, seguidas pela mucosa alveolar, pela língua, pelo lábio, pelo palato, pelo assoalho de boca e pela gengiva $^{12}$.

Em relação ao tamanho da lesão, no presente estudo, $34,09 \%$ (quinze casos) apresentaram mais do que $20 \mathrm{~mm}$ no seu maior diâmetro, $59,09 \%$ (vinte e seis casos) apresentaram menos do que $20 \mathrm{~mm}$ e $6,81 \%$ (três casos) dos laudos histopatológicos não apresentavam essa informação. Outro estudo utilizou o sistema de clas- sificação proposto por Axéll et al. ${ }^{13}$, de forma que $50 \%$ das lesões foram classificadas como L1 $(<2 \mathrm{~cm}), 38,9 \%$ como L2 $(2-4 \mathrm{~cm})$, e o restante $(11,1 \%)$, como L3 $(>4 \mathrm{~cm})^{8}$.

Histopatologicamente, no presente estudo, a acantose associada à hiperceratose/hiperparaceratose/hiperortoceratose foi predominante (vinte e oito casos - 63,6\%), e a displasia epitelial apenas esteve presente em $6,8 \%$ da amostra (três casos). $O$ epitélio displásico ou carcinoma invasivo são encontrados de 5\% a 25\% das amostras biopsiadas ${ }^{3}$. Em diversos estudos e localizações, a taxa de transformação da leucoplasia em lesão maligna varia entre 0,6 e $18 \%$. Os últimos estudos sugerem um potencial de transformação maligna de 4\%. Embora apresente a displasia como um fator para a cancerização, isso não quer dizer que, obrigatoriamente, a lesão vai malignizar, assim como nem todas as lesões que não apresentam epitélio displásico estão livres da malignizaçã $0^{3,10,11,14,15}$.

$\mathrm{Na}$ presente investigação, não houve diferenças estatisticamente significativas $(p>0,05)$ ao se relacionar o número médio de NORs por núcleo e as características clínicas ou histopatológicas da leucoplasia. Tal achado corrobora o trabalho de Epivatianos ${ }^{16}$, que estudou uma possível correlação entre a localização do carcinoma epidermóide bucal e a contagem de AgNORs, não observando diferenças estatisticamente significativas. Assim, o autor afirma que o número de NORs não é indicativo de prognóstico dos carcinomas epidermóides bucais em relação à localização.

No entanto, um estudo analisou 34 pacientes com carcinoma adenoide cístico (CAC) para verificar a quantificação das AgNORs, os pacientes foram divididos em dois grupos. O grupo um foi composto por vinte pacientes sem metástases, dos quais dezesseis estavam livres da doença, e o grupo dois foi composto por quatorze pacientes com metástases entre os quais deze morreram por causa do tumor. Todos os pacientes de grupo dois tinham a contagem de AgNOR superior a quatro enquanto que $65 \%$ dos pacientes do grupo um apresentou a média de contagem de AgNOR inferior a quatro. A análise estatística mostrou que a contagem de AgNOR no grupo dois foi significativamente maior do que no grupo um, sugerindo, assim, que a técnica AgNOR pode ser um método útil para a avaliação prognóstica do CAC e pode, portanto, ser utilizada para planejar o tratamento ${ }^{17}$.

Ahmed e Babiker ${ }^{18}$ (2009) ao avaliarem duzentos voluntários, dentre os quais 75 eram usuários de uma forma de tabaco mastigável usado no Sudão, 75 eram fumantes de cigarros e cinquenta não usavam tabaco, verificaram que a atipia celular foi significativamente mais elevada e o número de NORs por núcleo foi maior nos usuários de tabaco se comparado aos não usuários. Segundo esses autores, os componentes do tabaco, especialmente as nitrosaminas, podem estimular as células epiteliais a sofrerem 
diferenciação escamosa ou alterações morfológicas celulares que podem conduzir à malignidade.

Ao relacionar a graduação histológica dos carcinomas epidermóides bucais com o número médio de NORs, um estudo demonstrou que as lesões com seis ou mais NORs por núcleo podem ter um comportamento mais agressivo e apresentar um prognóstico pior ${ }^{18}$. No presente estudo, a média de NORs foi de 2,63, que, segundo Sano et al. $(1991)^{19}$, não representa comportamento agressivo.

Embora não se tenha obtido resultados estatisticamente significativos ao se relacionar o número médio de NORs das leucoplasias bucais com suas características clínicas ou histopatológicas, deve-se considerar que autores como Vahuhala ${ }^{17}$; Ahmed e Babiker ${ }^{18}$, mencionam ser o método AgNOR de grande valia na determinação do prognóstico de lesões bucais. Igualmente, deve-se considerar que ao se ampliar a amostra estudada, os resultados obtidos poderiam ser diferentes, tornando-se significativos. Assim, sugere-se que mais estudos clínico-histoquímicos, tanto transversais quanto longitudinais, sejam realizados a fim de se comprovar a efetividade do método testado.

\section{Conclusão}

A leucoplasia bucal acomete principalmente homens acima dos 60 anos de idade, brancos e fumantes. A maior parte das lesões apresenta tempo de evolução de até 1 ano, localizando-se na língua. A avaliação da atividade proliferativa das leucoplasias bucais por meio da contagem das NORs não mostrou relação com suas características clínicas ou histopatológicas.

\section{Agradecimento}

Os autores agradecem à Universidade de Passo Fundo por concessão de bolsa de iniciação científica (PIBIC/UPF) à acadêmica Angélica Zanata.

\section{Abstract}

Leukoplakia is considered a major carcinogenic oral lesion. Objective: To conduct an epidemiological study of oral leukoplakia cases registered at the Histopathology Diagnostic Service of the Institute of Biological Sciences, University of Passo Fundo (SDH/ICB-UPF), between 1989 and 2012, applying to such cases the AgNOR method for the assessment of their cell proliferative activity. Materials and method: The work consisted of a transversal epidemiological and histochemical study of 44 cases of leukoplakia. After diagnostic validation, and tabulation of clinical and histopathological data of leukoplakia cases, new histological sections $(3 \mu \mathrm{m})$ of lesions were impregnated by silver by the AgNOR method. The slides obtained were photographed in immersion, and 100 cells' NORs were counted for each case. This procedure was performed with the help of the Image Tool ${ }^{T M}$ software. Results were compared considering the different clinical and histopathological features of leukoplakia cases. Results: The most prevalent age group was from 61 to 76 years (19 cases - 43.2\%), 22 patients were men $(50 \%)$, and the predominant ethnic group was Caucasian (36 cases - 81.8\%). Regarding harmful habits, smoking was reported in 23 cases (52.3\%). When comparing the average number of NORs from cases of leukoplakia with different clinical features of the lesions, there was no statistically significant relation. Conclusion: It is concluded that leukoplakia primarily affects Caucasian men over 60 years old, who are smokers. Moreover, the sample studied showed that the relation of cell proliferative activity of oral leukoplakia with clinical/histopathological features of lesions was not statistically significant.

Keywords: Oral leukoplakia. AgNOR. Proliferative activity.

\section{Referências}

1. WHO. Colaboration Centre for Oral Precancerous Lesions. Definition of leukoplakia and related lesions: an aid to studies on oral precancer. Oral Surg Oral Med Oral Pathol. 1978;46(4):518-539.

2. Neville BW, Damm DD, Allen CM, Bouquot JE. Patologia epitelial. In: . Patologia Oral \& Maxilofacial. 3. ed. Rio de Janeiro: Guanabara Koogan; 2009.

3. Silva ICO, Cavalho ATD, Silva LBO, Nagahama MCVFB. Leucoplasia: uma revisão de literature / Leukoplakia: a literature revision. RGO 2007;55(3):287-89.

4. Derenzini M, Ceccarelli C, Santini D, Taffurelli M, Treté D. The prognostic value of the AgNOR parameter in human breast cancer depends on the pRB and p53 status. J Clin Pathol 2004;57:755-761.

5. Ray JG, Chattopadhyay A, Caplan DJ. Usefulness of AgNOR counts in diagnosing epithelial dysplasia. J Oral Pathol Med 2003;32:71-76.

6. Nunes FD, Pinto Jr, DS, Araújo NS, Araújo VC. Morphological study and optimization of the AgNOR technique. In: Meeting of the Brazilian Society for Dental Research: 1991; São Paulo; Proceedings of SBPqO; 1991. p. 15.

7. Goés C, Weyll B, Sarmento VA, Ramalho LMP. Diagnóstico diferencial e manejo da leucoplasia bucal - caso clínico: acompanhamento de 4 anos. RGO 2007;55(1):95-100.

8. Vázquez AR, Fernández GF, Gándara VP, Reboiras LD, García GA, Gándara RJ. Correlation between clinical and pathologic diagnosis in oral leukoplakia in 54 patients. Med Oral Patol Oral Cir Bucal 2010;15(6):832-8.

9. Mishra M, Mohanty J, Sengupta S, Tripathy S. Epidemiological and clinicopathological study of oral leukoplakia. Indian J Dermatol Venereol Leprol 2005;71:161-5.

10. Pedreira EM, Cardoso CL, Barroso EC, Santos JAS, Fonseca FP, Taveira LAA. Epidemiological and oral manifestations of HIV-positive patients in a specialized service in Brazil. J Appl Oral Sci 2008;16(6):369-75.

11. Brzak BL, Mravak-stipetic M, Canjuga I, Baricevic M, Balicevic D, Sikora M, et al. The Frequency and Malignant Transformation Rate of Oral Lichen Planus and Leukoplakia - A Retrospective Study. Coll Antropol 2012;3:773-777.

12. Gabriel JG, Cherubini K, Yurgel LS, Figueiredo MAZ. Considerações gerais e abordagem clínica na leucoplasia oral. Rev Bras Patol Oral 2004;3(4):187-194. 
13. Axéll T, Pindborg JJ, Smith CJ, Van Der Waal I. Oral white lesions with special reference to precancerous and tobaccorelated lesions: conclusions of an international symposium held in Uppsala, Sweden, May 18-21 1994. International Collaborative Group on Oral White Lesions. J Oral Pathol Med 1996;25:49-54.

14. Batista Castro Z, Rodríguez Pérez I, Miranda Tarragó JD, Rengifo Calzado E, Rodríguez Obaya T, Fernández-Jiménez ME. Caracterización histopatológica de la leucoplasia bucal en La Habana durante diez años. Rev Cubana Estomatol. 2011;48(4):31-3

15. Santana JC. Atlas de patología del complejo bucal. La Habana: Editorial de Ciencias Médicas; 2010.

16. Epivatianos AA. Evaluation of the nucleolar organizer region associated proteins in oral squamous cell carcinoma. Annals of Dentistry 1994;53(1):33-36.

17. Vuhahula EAM. Prognostic value of argyrophilic nucleolar organizer regions (Agnor) count in adenoid cystic carcinoma of salivary glands. Pathol Int 1994;44(5):368-373.

18. Ahmed HG, Babiker AEA. Assessment of cytological atypia, AgNOR and nuclear area in epithelial cells of normal oral mucosa exposed to toombak and smoking. Rare Tumors 2009;1:50-52.

19. Sano K, Takahashi H, Fujita S, Inokuchi T, Pe MB, Okabe $\mathrm{H}$, et al. Prognostic implication of silver-binding nucleolar organizer regions (Agnors) in oral squamous cell carcinoma. J Oral Pathol Med 1991;20(2):53-6.

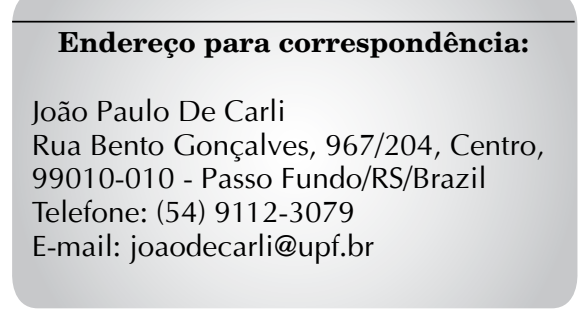

Recebido: 05/05/2014. Aceito: 01/12/2014. 